\title{
International Agency for Research on Cancer
}

\author{
IARC Monographs Volume 112: evaluation of \\ five organophosphate insecticides and herbicides
}

Lyon, France, 20 March 2015 - The International Agency for Research on Cancer (IARC), the specialized cancer agency of the World Health Organization, has assessed the carcinogenicity of five organophosphate pesticides. A summary of the final evaluations together with a short rationale have now been published online in The Lancet Oncology, and the detailed assessments will be published as Volume 112 of the IARC Monographs.

\section{What were the results of the IARC evaluations?}

The herbicide glyphosate and the insecticides malathion and diazinon were classified as probably carcinogenic to humans (Group 2A).

The insecticides tetrachlorvinphos and parathion were classified as possibly carcinogenic to humans (Group 2B).

\section{What was the scientific basis of the IARC evaluations?}

The pesticides tetrachlorvinphos and parathion were classified as possibly carcinogenic to humans (Group 2B) based on convincing evidence that these agents cause cancer in laboratory animals.

For the insecticide malathion, there is limited evidence of carcinogenicity in humans for non-Hodgkin lymphoma and prostate cancer. The evidence in humans is from studies of exposures, mostly agricultural, in the USA, Canada, and Sweden published since 2001. Malathion also caused tumours in rodent studies. Malathion caused DNA and chromosomal damage and also disrupted hormone pathways.

For the insecticide diazinon, there was limited evidence of carcinogenicity in humans for non-Hodgkin lymphoma and lung cancer. The evidence in humans is from studies of agricultural exposures in the USA and Canada published since 2001. The classification of diazinon in Group 2A was also based on strong evidence that diazinon induced DNA or chromosomal damage.

For the herbicide glyphosate, there was limited evidence of carcinogenicity in humans for non-Hodgkin lymphoma. The evidence in humans is from studies of exposures, mostly agricultural, in the USA, Canada, and Sweden published since 2001. In addition, there is convincing evidence that glyphosate also can cause cancer in laboratory animals. On the basis of tumours in mice, the United States Environmental Protection Agency (US EPA) originally classified glyphosate as possibly carcinogenic to humans (Group C) in 1985. After a re-evaluation of that mouse study, the US EPA changed its classification to evidence of non-carcinogenicity in humans (Group E) in 1991. The US EPA Scientific Advisory Panel noted that the re-evaluated glyphosate results were still significant using two statistical tests recommended in the IARC Preamble. The IARC Working Group that conducted the evaluation considered the significant findings from the US EPA report and several more recent positive results in concluding that there is sufficient evidence of carcinogenicity in experimental animals. Glyphosate also caused DNA and chromosomal damage in human cells, although it gave negative results in tests using bacteria. One study in community residents reported increases in blood markers of chromosomal damage (micronuclei) after glyphosate formulations were sprayed nearby.

\section{How are people exposed to these pesticides?}

Tetrachlorvinphos is banned in the European Union. In the USA, it continues to be used on livestock and companion animals, including in pet flea collars. No information was available on use in other countries.

Parathion use has been severely restricted since the 1980s. All authorized uses were cancelled in the European Union and the USA by 2003. 


\section{IARC Monographs Volume 112: evaluation of five organophosphate insecticides and herbicides}

Malathion is currently used in agriculture, public health, and residential insect control. It continues to be produced in substantial volumes throughout the world. Workers may be exposed during the use and production of malathion. Exposure to the general population is low and occurs primarily through residence near sprayed areas, home use, and diet.

Diazinon has been applied in agriculture and for control of home and garden insects. Production volumes have been relatively low and decreased further after 2006 due to restrictions in the USA and the European Union. Only limited information was available on the use of these pesticides in other countries.

Glyphosate currently has the highest global production volume of all herbicides. The largest use worldwide is in agriculture. The agricultural use of glyphosate has increased sharply since the development of crops that have been genetically modified to make them resistant to glyphosate. Glyphosate is also used in forestry, urban, and home applications. Glyphosate has been detected in the air during spraying, in water, and in food. The general population is exposed primarily through residence near sprayed areas, home use, and diet, and the level that has been observed is generally low.

\section{What do Groups $2 A$ and $2 B$ mean?}

Group 2A means that the agent is probably carcinogenic to humans. This category is used when there is limited evidence of carcinogenicity in humans and sufficient evidence of carcinogenicity in experimental animals. Limited evidence means that a positive association has been observed between exposure to the agent and cancer but that other explanations for the observations (called chance, bias, or confounding) could not be ruled out. This category is also used when there is limited evidence of carcinogenicity in humans and strong data on how the agent causes cancer.

Group 2B means that the agent is possibly carcinogenic to humans. A categorization in Group 2B often means that there is convincing evidence that the agent causes cancer in experimental animals but little or no information about whether it causes cancer in humans.

\section{Why did IARC evaluate these pesticides?}

The IARC Monographs Programme has evaluated numerous pesticides, some as recently as 2012 (anthraquinone, arsenic and arsenic compounds). However, substantial new data are available on many pesticides that have widespread exposures. In 2014, an international Advisory Group of senior scientists and government officials recommended dozens of pesticides for evaluation. Consistent with the advice of the Advisory Group, the recent IARC meeting provided new or updated evaluations on five organophosphate pesticides.

\section{How were the evaluations conducted?}

The established procedure for Monographs evaluations is described in the Programme's Preamble. Evaluations are performed by panels of international experts, selected on the basis of their expertise and the absence of real or apparent conflicts of interest. For Volume 112, a Working Group of 17 experts from 11 countries met at IARC on 3-10 March 2015 to assess the carcinogenicity of tetrachlorvinphos, parathion, malathion, diazinon, and glyphosate. The in-person meeting followed nearly a year of review and preparation by the IARC secretariat and the Working Group, including a comprehensive review of the latest available scientific evidence. According to published procedures, the Working Group considered "reports that have been published or accepted for publication in the openly available scientific literature" as well as "data from governmental reports that are publicly available". The Working Group did not consider summary tables in online supplements to published articles, which did not provide enough detail for independent assessment.

\section{What are the implications of the IARC evaluations?}

The Monographs Programme provides scientific evaluations based on a comprehensive review of the scientific literature, but it remains the responsibility of individual governments and other international organizations to recommend regulations, legislation, or public health intervention.

Media inquiries: please write to com@iarc.fr. Thank you. 